\title{
Preliminary study of beta-blocker therapy on modulation of interleukin-33/ST2 signaling during ventricular remodeling after acute myocardial infarction
}

\author{
Jinggang Xia ${ }^{1, *}$, Yang $\mathrm{Qu}^{2, *}$, Chunlin $\mathrm{Yin}^{1}$, Dong $\mathrm{Xu}^{1}$ \\ ${ }^{1}$ Department of Cardiology, Xuanwu Hospital, Capital Medical University, Beijing, China \\ ${ }^{2}$ Department of Pathology, Beijing Chest Hospital, Capital Medical University, Beijing, China
}

\begin{abstract}
Background: This study aimed to evaluate the role of $\beta$-blocker therapy on modulating interleukin (IL)-33/ST2 (interleukin-1 receptor-like 1) signaling during ventricular remodeling related to heart failure (HF) after acute myocardial infarction (AMI).

Methods: Sprague-Dawley rats that survived surgery to induce AMI were randomly divided into the placebo group and the $\beta$-blocker treatment group. A sham group was used as a control. Left ventricular (LV) function variables, the myocardial infarct size, fibrosis and IL-33/ST2 protein expression was measured.

Results: Compared with the placebo group, $\beta$-blocker treatment significantly improved $L V$ function and reduced infarct size $(p<0.05)$. There was higher protein expression of $I L-33(p<0.05)$ and sST2 $(p<0.05)$, as well as higher expression of fibrosis $(p<0.05)$, compared to the sham group. Notably, the high expression of cardioprotective IL-33 was not affected by $\beta$-blocker treatment $(p>0.05)$, however, treatment with $\beta$-blocker enhanced IL-33/ST2 signaling, with lower expression of sST2 $(p<0.05)$ and significantly attenuated fibrosis $(p<0.05)$.

Conclusions: Our study suggested that $\beta$-blocker therapy might play a beneficial role in the modulation of IL-33/ST2 signaling during ventricular remodeling. These results may be helpful in identifying IL-33/ST2 systems as putative $\beta$-blocker targets at an early stage after AMI. (Cardiol J 2017; 24, 2: 188-194) Key words: beta-blocker, IL-33/ST2, ventricular remodeling, acute myocardial infarction
\end{abstract}

\section{Introduction}

Heart failure (HF) and acute myocardial infarction (AMI) are the most common causes of morbidity and mortality worldwide [1]. The loss of cardiac function after AMI drives specific cardiac remodeling and hypertrophy processes in order to preserve cardiac output, however, cardiac remodeling and hypertrophy processes lead to further deterioration in cardiac function [2]. During the past several decades, a large body of evidence has suggested that $\beta$-blocker therapy can reduce HF-related morbidity and mortality [3]. While these studies have shown that $\beta$-blockers can improve left ventricular (LV) remodeling [4], the protective mechanisms are not well understood.

ST2 is an interleukin-1 (IL-1) receptor family member with both soluble (sST2) and transmembrane (ST2L) isoforms. Myocardial fibrosis and hypertrophy are attenuated through the interaction between IL-33 and ST2L [5-8]. The role of sST2 in remodeling seems to be mediated by its effect

Address for correspondence: Chunlin Yin, PhD or Dong Xu, PhD, Department of Cardiology, Xuanwu Hospital, Capital Medical University, Beijing, 100053, China, tel: 8613621041267, fax: 86-10-83198252, Chunlin Yin: e-mail: yinclmail@gmail.com; DongXu: e-mail: xudheart@aliyun.com

*The first two authors contributed equally to this study.

Received: 11.07.2016 Accepted: 08.10.2016 
on its primary ligand, IL-33, which itself is also synthesized when cardiac fibroblasts are mechanically stretched. IL-33 has been shown to inhibit cardiomyocyte hypertrophy, fibrosis, and apoptosis. In contrast, sST2 acts as a decoy receptor that neutralizes IL-33. In addition, sST2 impairs the cardioprotective role of the IL-33/ST2L signaling pathway, resulting in cardiac hypertrophy and fibrosis. Several studies have shown that sST2 levels can significantly predict LV remodeling and can strongly predict the adverse outcomes in patients with HF [9]. Therefore, sST2 may be a promising prognostic biomarker in patients with established HF after AMI.

Given the relationship between sST2 and HF, we hypothesized that an intersection between $\beta$-blocker therapy and the IL-33/ST2L signaling pathway may exist. The current study aimed to evaluate the role of $\beta$-blocker therapy in modulating IL-33/ST2 signaling during ventricular remodeling related to $\mathrm{HF}$ after AMI.

\section{Methods}

\section{Study protocol}

The current study followed the Guide for the Use and Care of Laboratory Animals published by the United States National Institutes of Health. The research was approved by the Animal Research Councils and the Ethics Council, Health Center, Xuanwu Hospital, Capital Medical University, China.

Fifty healthy male pathogen-free SpragueDawley rats $(280 \pm 30 \mathrm{~g})$ from the Experimental Animal Center of Xuanwu Hospital, Capital Medical University were used in this study. The AMI model was established by occlusion of the left anterior descending artery in 42 rats, as described previously [10]. At the same time point, according to two previous studies [11, 12], we estimated that a sample of 8 rats in the sham group was appropriate when considering cost and ethics. The 8 rats received the same operation without ligation of the left anterior descending coronary artery. Anesthesia was induced by an intraperitoneal injection of pentobarbital sodium solution $(2 \%)$. The rats were ventilated mechanically (oxygen, $1 \mathrm{~L} / \mathrm{min}$ ). Twenty-four hours later, only 30 rats with AMI had survived, but all 8 sham rats had survived. The 30 rats with AMI were randomized in a 1:1 ratio into the placebo group and the $\beta$-blocker treatment group as follows: (1) The placebo group, the AMI rats receiving water by gavage $(\mathrm{n}=15)$ and (2) The $\beta$-blocker treatment group, the AMI rats receiving bisoprolol (from Merck KGaA, Darmstadt, Germany) ( $\mathrm{n}=15 ; 10 \mathrm{mg} / \mathrm{kg} /$ day in drinking water for 4 weeks); (3) In addition, a sham group (without AMI) received water by gavage $(n=8)$. After a 4-week study period, echocardiography was performed and the respective parameters were obtained. Subsequently, the $30 \mathrm{AMI}$ rats and the 8 sham rats were euthanized, and the hearts were harvested. The myocardial infarct size was measured, and western blot assays of the infarcted area were performed.

\section{Echocardiographic measurements}

Left ventricular function variables were evaluated by using echocardiography in the three groups of rats anesthetized with pentobarbital sodium solution (2\%). A Vevo-770 small animal ultrasound (VisualSonics Inc., Toronto, Canada) with a $17.5-\mathrm{MHz}$ probe frequency was used to assess the cardiac characteristics in a noninvasive manner. The Mmode images were taken through the papillary muscle level short axis. The thicknesses of the LV end-diastolic anterior wall (LVEDAW), LV end-diastolic posterior wall (LVEDPW), LV enddiastolic diameter (LVEDD), and LV end-systolic diameter (LVESD) were measured. According to the American Standards of Echocardiography, the variables of three to five consecutive cardiac cycles were observed and measured. The ejection fraction (EF) and fractional shortening (FS) were calculated according to a previous report [13]. All images were analyzed by Vevo-770 software (VisualSonics Inc.).

\section{Infarct size}

At the end of week $4(\mathrm{w} 4)$, the hearts were harvested. Five rats in the $\beta$-blocker treatment group and 5 rats in the placebo group underwent myocardial infarct size measurement by 2,3 , 5 -triphenyltetrazolium chloride (TTC) staining. Myocardial infarct size in the sham group was not measured. The surgical procedure was based on a previous research report [14]. The hearts were rapidly excised, atria were removed, and ventricles were sliced transversely into five cross slices with uniform thickness. And then the slices were stained at $37^{\circ} \mathrm{C}$ for $30 \mathrm{~min}$ with $1 \%$ TTC, which stains only the viable myocardium. The white region indicated an infarcted myocardium (area of necrosis, $\mathrm{AN}$ ), the light red region indicated an ischemic myocardium, and the blue region indicated a normal myocardium. The ratio of the infarcted area and the area at risk (the sum of the ischemic and infarcted areas, AAR), which is AN/AAR, was used 
Table 1. Effect of $\beta$-blocker treatment on echocardiographic data on the cardiac structure and function in rats following acute myocardial infarction ( $\mathrm{n}=15$ each for the placebo group and the $\beta$-blocker treatment group; $\mathrm{n}=8$ for the sham group).

\begin{tabular}{lccc}
\hline Parameter & Sham group & Placebo group & Beta-blocker \\
\hline LV end-diastolic anterior wall [mm] & $1.7 \pm 0.2$ & $0.5 \pm 0.1^{*}$ & $0.8 \pm 0.1^{\#}$ \\
LV end-systolic diameter [mm] & $3.3 \pm 0.2$ & $4.5 \pm 0.3^{*}$ & $4.1 \pm 0.2^{\#}$ \\
LV end-diastolic diameter [mm] & $7.1 \pm 0.3$ & $9.2 \pm 0.4^{*}$ & $8.6 \pm 0.3^{\#}$ \\
LV end-diastolic posterior wall [mm] & $1.7 \pm 0.1$ & $1.6 \pm 0.1$ & $1.6 \pm 0.2$ \\
Ejection fraction [\%] & $75.8 \pm 4.5$ & $48.5 \pm 7.7^{*}$ & $62.8 \pm 5.6^{\#}$ \\
Fractional shortening [\%] & $49.3 \pm 2.7$ & $31.5 \pm 3.3^{*}$ & $40.8 \pm 3.8^{\#}$ \\
\hline
\end{tabular}

Continuous variables are presented as mean \pm standard deviation. *Significant difference when compared with the sham group. ${ }^{*}$ Significant difference when compared with the placebo group; LV - left ventricular

to represent the extent of the infarction. AAR/LV was used to represent the extent of the AAR. AN, $\mathrm{AAR}$, and LV were measured by Image-ProPlus analysis software (version 4.1; Media Cybernetics, Rockville, MD, USA).

Western blot assay of IL-33, sST2, Col I, Col III, and TIMP-1

At the end of $\mathrm{w} 4$, the AN was collected and total protein was extracted as described previously [15]. The remaining 10 rats in $\beta$-blocker treatment and placebo groups and 8 rats in the sham group were designated for Western blot assay. The tissue for protein extraction in the sham group was from the LV anterior wall near the apex. The location was similar with that in the control and $\beta$-blocker treatment groups. The extracted protein $(200 \mu \mathrm{g})$ was added to an $8 \%$ SDS-PAGE separating gel and transferred to a nitrocellulose membrane (Millipore, Bedford, MA, USA). Following blocking, the membrane was incubated at room temperature for $4 \mathrm{~h}$ with one of the following primary antibodies: IL-33, sST2, collagen type I (Col I), collagen type III (Col III), and tissue inhibitor of metalloproteinase-1 (TIMP-1) (all diluted 1:500) from Santa Cruz Biotechnology (Santa Cruz, CA, USA). The antibodytagged membranes were probed with a secondary antibody solution consisting of either a 1:1,000 dilution of horseradish peroxidase-conjugated rabbit anti-rat IgG (for IL-33, sST2) or a 1:1,000 dilution of horseradish peroxidase-conjugated goat anti-rabbit IgG (for Col I, Col III, TIMP-1, and glyceraldehyde phosphate dehydrogenase (GAPDH) from Promega (Madison, WI, USA) for $1 \mathrm{~h}$ at room temperature. The integrated optical density (mean optical density $x$ area) was detected by Image-ProPlus analysis software. The ratio of the target protein and GAPDH reflected the relative level of the target protein.

\section{Statistical analysis}

Continuous variables were presented as mean \pm standard deviation (SD), and differences between groups were compared by one-way analysis of variance (ANOVA) followed by Student-NewmanKeuls post hoc analysis. Categorical variables between groups were compared by Fisher's exact test. The value of $p<0.05$ was considered statistically significant. Statistics were analyzed using SPSS software version 19 (SPSS Inc., Chicago, IL, USA).

\section{Results}

All 8 rats in the sham group survived surgery. Four weeks after induction of AMI, 30 rats survived and 12 rats died. One rat died during anesthesia, 7 rats died of ventricular arrhythmia, 1 rat died of pneumothorax, and 3 rats died of HF during the procedure. The 30 rats that survived were randomly divided into the placebo group or the $\beta$-blocker treatment group.

\section{Echocardiography}

At w4 after induction of AMI, the placebo group had significantly higher LVEDD and LVESD values ( $p<0.05$ vs. the sham group), as well as significantly lower LVEDAW, FS, and EF values $(\mathrm{p}<0.05$ vs. the sham group), however, the placebo rats did not differ from the rats in the sham group in terms of the LVEDPW thickness ( $p>0.05$ ).

Beta-blocker treatment significantly decreased the LVEDD and LVESD values ( $p<0.05$ vs. the placebo group) and significantly increased the LVEDAW, FS, and EF values ( $p<0.05$ vs. the placebo group), however, the LVEDPW thickness did not change significantly after $\beta$-blocker treatment ( $p>0.05$ vs. the placebo group; Table 1$)$. 


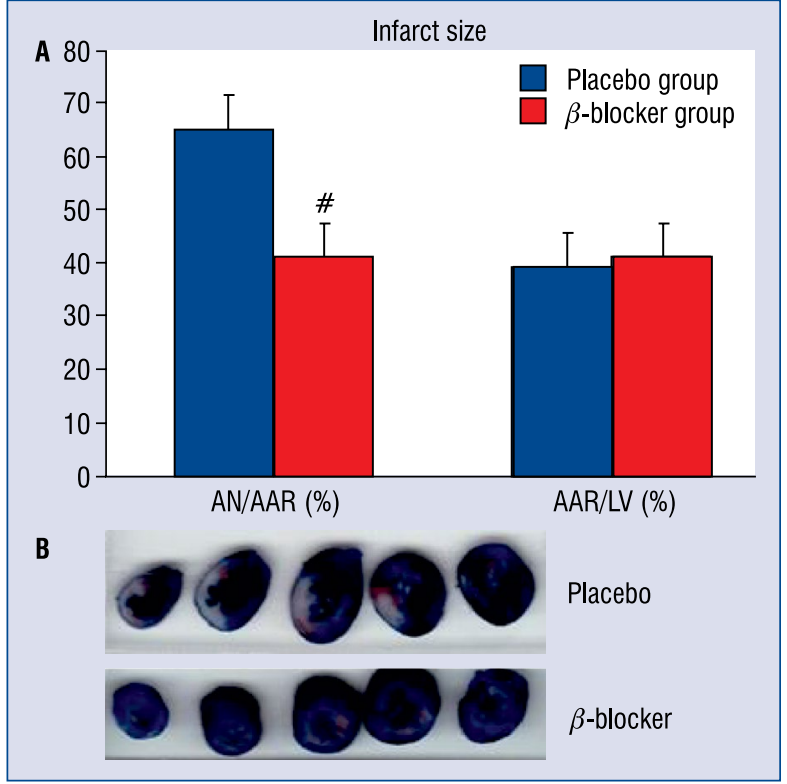

Figure 1. A. Comparisons of AN/AAR and AAR/LV between the placebo and $\beta$-blocker treatment groups $(n=5$ per group). The blue bars indicate placebo group and the red bars indicate $\beta$-blocker treatment group. Variables are presented as mean \pm standard deviation and compared by one-way ANOVA. \#Significant difference compared to the placebo group. The ratio of the infarcted area and the area at risk (the sum of the ischemic and necrotic areas) (AN/AAR) was used to represent the extent of the infarction. AAR/LV was used to represent the extent of the AAR; AN - area of necrosis; AAR area at risk; LV — area of left ventricle; $B$. Staining of the myocardium with triphenyltetrazolium chloride. Ventricles were sliced transversely into five cross slices with uniform thickness. From right to left: heart base to apex. The white region indicates an infarcted myocardium, the light red region indicates an ischemic myocardium, and the blue region indicates a normal myocardium.

\section{Infarct size}

The placebo group showed an obvious myocardial infarction (AN/AAR, $65.23 \pm 6.46 \%$ ). Compared with the placebo group, the AN/AAR ratio of the $\beta$-blocker treatment group (AN/AAR, $48.96 \pm$ $\pm 6.22 \%)$ was significantly lower $(\mathrm{p}<0.05)$. AAR/ $/ \mathrm{LV}$ was similar between the placebo group (41.26 \pm $\pm 6.12 \%)$ and the $\beta$-blocker treatment group $(40.96 \pm$ $\pm 5.92 \%)(\mathrm{p}>0.05$; Fig. 1).

\section{IL-33/ST2 signaling pathway}

The IL-33/ST2 signaling pathway was evaluated by analyzing the protein expression of cardioprotective IL-33 and sST2, as shown in Figure 2. In the infarcted myocardium, there was higher protein expression of IL-33 $(\mathrm{p}<0.05)$ and sST2

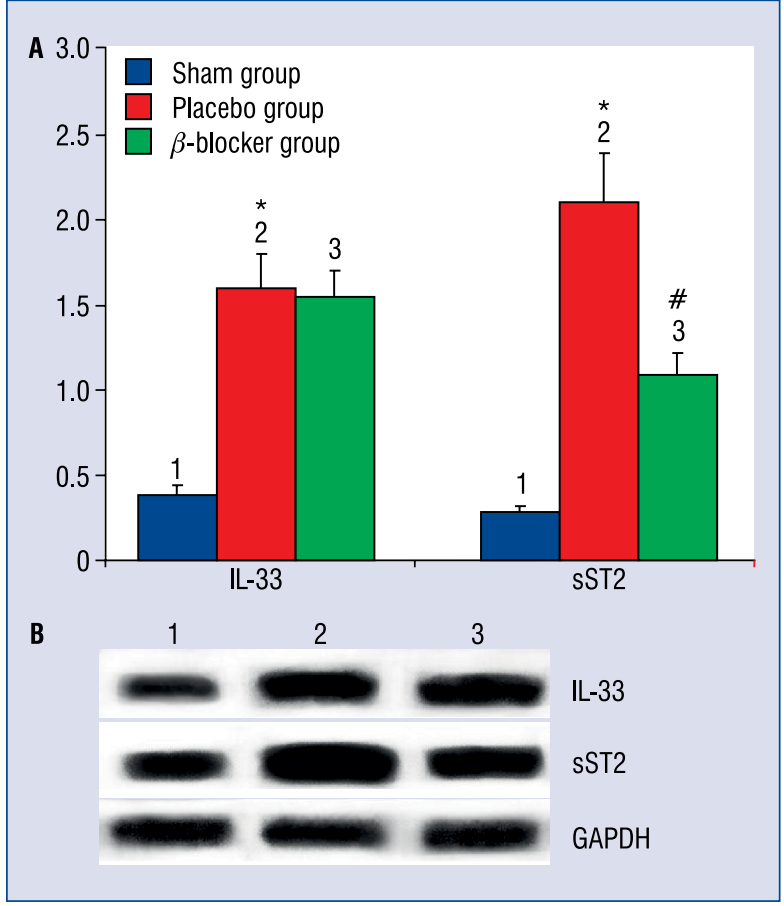

Figure 2. Protein expression of interleukin (IL)-33 and sST2 in the three groups; A. Comparisons of IL-33 and sST2 among the three groups ( 10 rats in $\beta$-blocker treatment group, 10 in placebo group and 8 in sham group). The blue bars indicate sham group, the red bars indicate placebo group and green bars indicate $\beta$-blocker treatment group. Variables are presented as mean \pm \pm standard deviation and compared by one-way ANOVA; *Significant difference compared with the sham group; \#Significant difference compared with the placebo group; B. Representative images of the protein expression of IL-33, sST2, and GAPDH. Note: GAPDH is a reference; 1 - sham group; 2 - placebo group; 3 $\beta$-blocker treatment group; GAPDH - glyceraldehyde phosphate dehydrogenase.

( $p<0.05)$, compared to the sham group. Notably, the high protein expression of the cardioprotective IL-33 was not affected by $\beta$-blocker treatment $(\mathrm{p}>0.05)$, however, the sST2 levels were significantly reduced by $\beta$-blocker treatment $(\mathrm{p}<0.05)$.

\section{Fibrosis}

Fibrosis was evaluated by analyzing the protein expression of Col I, Col III, and TIMP-1. As shown in Figure 3, in the infarcted area, there was higher expression of Col I, Col III, and TIMP- 1 in the placebo group $(\mathrm{p}<0.05)$, compared to the sham group. Compared to the placebo group, $\beta$-blocker treatment was associated with lower expression of Col I, Col III, and TIMP-1 $(\mathrm{p}<0.05)$. 


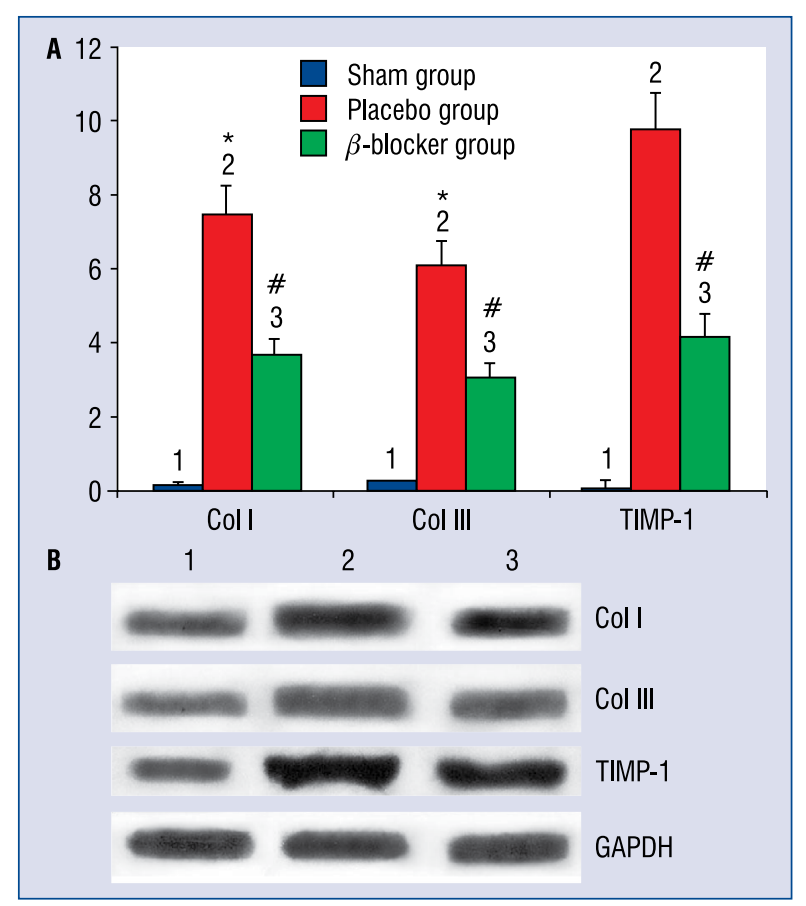

Figure 3. Protein expression of collagen type I (Col I), collagen type III (Col III), and tissue inhibitor of metalloproteinase (TIMP)-1 in the three groups; A. Comparisons of Col I, Col III, and TIMP-1 among the three groups (10 rats in $\beta$-blocker treatment group, 10 in placebo group and 8 in sham group). The blue bars indicate sham group, the red bars indicate placebo group and green bars indicate $\beta$-blocker treatment group. Variables are presented as mean \pm standard deviation and compared by one-way ANOVA; *Significant difference compared with the sham group; \#Significant difference compared with the placebo group; B. Representative images of the protein expression of Col I, Col III, TIMP-1, and GAPDH; Note: GAPDH is a reference; 1 - sham group; 2 - placebo group; $3-\beta$-blocker treatment group; GAPDH - glyceraldehyde phosphate dehydrogenase.

\section{Discussion}

Due to the high prevalence of $\beta$-blocker prescriptions in patients with cardiovascular disease, there is significant interest in elucidating the mechanisms contributing to the clinical benefit of $\beta$-blocker therapy. Similarly, there have been efforts to increase the understanding of the role of sST2 in the pharmacology of $\beta$-blocker therapy. However, the contribution of IL-33/ST2 signaling in ventricular remodeling related to $\mathrm{HF}$ after AMI remains unknown. To the best of our knowledge, this is the first study to show an interaction between $\beta$-blocker therapy and IL-33/ST2 signaling. Notably, we demonstrated that $\beta$-blocker therapy modulates IL-33/sST2 signaling and cardiac ventricular remodeling, which correlate with markers of fibrosis. This signaling could be responsible for the cardioprotective effects of $\beta$-blocker therapy in AMI patients.

Heart failure is a very complex clinical syndrome due to structural or functional impairment of ejection of blood and/or ventricular filling [16]. Biomarkers are indispensable tools for diagnosis and prognostication in many kinds of cardiovascular diseases, and some have become standard of care [17]. Soluble ST2 is a novel biomarker that reflects inflammation, fibrosis, and cardiac stress, which is correlated with the pathophysiology of adverse myocardial remodeling and HF [18]. ST2 measurements in patients with either acute or chronic HF are now strongly recommended for additive risk stratification, according to the 2013 American College of Cardiology Foundation and American Heart Association joint guidelines [19]. However, the expected benefits of $\beta$-blocker therapy in those with elevated concentrations of sST2 have been elusive. The recent PROTECT study has shown that chronic systolic HF patients with lower ST2 levels are likely to get the most benefit from higher $\beta$-blocker doses [20]. While this report is encouraging, the putative link between ST2 and the cardioprotective effect of $\beta$-blocker therapy requires further clarification.

One of the critical factors in cardiac remodeling is excessive activation of the sympathetic nervous system. It contributes to the progression of $\mathrm{HF}$ through remodeling, thus providing a biomechanical explanation for the use of medications to inhibit these pathways to deter the progression of $\mathrm{HF}$ through inhibiting adverse remodeling [21, 22]. Over the past decade, several randomized clinical trials have shown that administration of $\beta$-blockers improves LV function and reduces morbidity and mortality in patients with both acute and chronic HF [23-26].

In the present study, we used an animal model of AMI to evaluate myocardial expression of IL-33 and sST2 at w 4 after $\beta$-blocker therapy. We found that compared with the sham group, the placebo group had significantly decreased LV function as shown by the higher LVESD and LVEDD values, as well as the lower LVEDAW, FS, and EF values. In the infarcted myocardium, compared with the sham group, the placebo group featured higher concentrations of IL-33 and sST2, as well as higher concentrations of Col I, Col III, and TIMP-1. The upregulation of IL-33 supports its participation in the protective response in the infarcted myocardium. 
IL-33 has been shown to inhibit cardiomyocyte hypertrophy, fibrosis, and apoptosis. The upregulation of sST2 supports the assumption that it acts as a decoy receptor that neutralizes IL-33 and impairs the cardioprotective role. The upregulation of IL-33 and sST2 correlates with cardiac markers of fibrosis in the setting of early remodeling after $\mathrm{AMI}$ and provides a plausible explanation for their measurable elevation. The effects on LV function and fibrosis in the placebo group and the effects on the IL-33/sST2 pathway are similar to the results reported previously [12]. Sanchez-Mas et al. [12] have reported that AMI upregulates the mRNA expression of sST2 and IL-33 in the infarcted myocardium and that the level of sST2 positively correlates with cardiac markers of fibrosis during the first week following AMI. In addition, the expression of sST2 is rapidly upregulated during the first 4 weeks, with levels correlating to the ongoing process of fibrosis. Moreover, Lax et al. [11] have reported that in the infarcted myocardium, compared with sham animals, the placebo group had higher concentrations of IL-33, sST2, and fibrosis markers.

We found that $\beta$-blocker therapy significantly decreased myocardial infarct size ratio compared with the placebo group. However, the area at risk ratio was similar between the $\beta$-blocker therapy and placebo groups. These data suggest that the ligation location of the left anterior descending artery was similar between $\beta$-blocker therapy treatment group and the placebo group in our study.

We also found that $\beta$-blocker therapy significantly improved LV function, reduced the infarct size, enhanced IL-33/ST2 signaling with lower expression of sST2, and attenuated the levels of Col I, Col III, and TIMP-1, compared with the placebo group. Notably, $\beta$-blocker therapy decreased the expression of sST2 and did not affect the upregulated level of IL-33. It supports the suggested protective role of IL-33 in response to AMI. Such a finding would suggest an overall protective enhancement of IL-33/ST2L signaling. On the whole, our findings would be helpful in identifying IL-33/ /ST2 systems as putative $\beta$-blocker targets at an early stage after AMI.

\section{Conclusions}

In conclusion, our results are novel and substantially extend the understanding of how $\beta$-blocker therapy modulates IL-33/ST2 signaling during ventricular remodeling related to $\mathrm{HF}$ after AMI. Some limitations of the present study include the following: (1) There was insufficient selection of cardiac remodeling markers and evaluation of the injured myocardium. Therefore, assessment of additional cardiac remodeling markers, such as inflammatory markers and apoptosis markers could have added additional value to the current study. (2) The relationship between IL-33/ST2 and $\beta$-blocker therapy could have been analyzed using a correlation analysis. (3) Additional in vitro assays could have supplemented the in vivo study to explore the direct actions of $\beta$-blocker therapy in cardiomyocytes. We will focus on these limitations in our future studies. However, our present findings suggest that $\beta$-blocker therapy might play a beneficial role in the regulation of IL-33/ST2 signaling during ventricular remodeling related to HF after AMI. Our study implies that the beneficial actions of $\beta$-blocker therapy on ventricular remodeling related to HF after AMI could be mediated by enhanced IL-33/ST2 signaling.

\section{Acknowledgements}

This research received no specific grant from any funding agency in the public, commercial, or not-for-profit sectors.

\section{Conflict of interest: None declared}

\section{References}

1. Hunt SA, Abraham WT, Chin MH, et al. American College of Cardiology Foundation, American Heart Association. 2009 Focused update incorporated into the ACC/AHA 2005 Guidelines for the Diagnosis and Management of Heart Failure in Adults A Report of the American College of Cardiology Foundation/American Heart Association Task Force on Practice Guidelines Developed in Collaboration With the International Society for Heart and Lung Transplantation. J Am Coll Cardiol. 2009; 53(15): e1-e90, doi: 10.1016/j.jacc.2008.11.013, indexed in Pubmed: 19358937.

2. Sharma S, Colangelo LA, Lima J, et al. Effects of Weight and Weight Change on Cardiac Remodeling Over 20 Years: The CARDIA (Coronary Artery Risk Development in Young Adults) Study. J Am Coll Cardiol. 2015; 65(22): 2463-2465, doi: 10.1016/j. jacc.2015.03.561, indexed in Pubmed: 26046743.

3. Cole GD, Patel SJ, Zaman N, et al. „Triple therapy” of heart failure with angiotensin-converting enzyme inhibitor, betablocker, and aldosterone antagonist may triple survival time: shouldn't we tell patients? JACC Heart Fail. 2014; 2(5): 545-548, doi: 10.1016/j.jchf.2014.04.012, indexed in Pubmed: 25301161.

4. Fukui M, Goda A, Komamura K, et al. Changes in collagen metabolism account for ventricular functional recovery following beta-blocker therapy in patients with chronic heart failure. Heart Vessels. 2016; 31(2): 173-182, doi: 10.1007/s00380-014-0597-1, indexed in Pubmed: 25351137.

5. Dieplinger B, Mueller T. Soluble ST2 in heart failure. Clin Chim Acta. 2015; 443: 57-70, doi: 10.1016/j.cca.2014.09.021, indexed in Pubmed: 25269091. 
6. Miller AM, Liew FY. The IL-33/ST2 pathway--A new therapeutic target in cardiovascular disease. Pharmacol Ther. 2011; 131(2): 179-186, doi: 10.1016/j.pharmthera.2011.02.005, indexed in Pubmed: 21356240.

7. Yin H, Li P, Hu F, et al. IL-33 attenuates cardiac remodeling following myocardial infarction via inhibition of the p38 MAPK and NF- $\kappa$ B pathways. Mol Med Rep. 2014; 9(5): 1834-1838, doi: 10.3892/mmr.2014.2051, indexed in Pubmed: 24626881.

8. Miller AM. Role of IL-33 in inflammation and disease. J Inflamm (Lond). 2011; 8(1): 22, doi: 10.1186/1476-9255-8-22, indexed in Pubmed: 21871091.

9. Bayes-Genis A, de Antonio M, Galán A, et al. Combined use of high-sensitivity ST2 and NTproBNP to improve the prediction of death in heart failure. Eur J Heart Fail. 2012; 14(1): 32-38, doi: 10.1093/eurjhf/hrr156, indexed in Pubmed: 22179033.

10. Liu Mi, Wang XR, Wang C, et al. Panax quinquefolium saponin attenuates ventricular remodeling after acute myocardial infarction by inhibiting chop-mediated apoptosis. Shock. 2013; 40(4): 339-344, doi: 10.1097/SHK.0b013e3182a3f9e5, indexed in Pubmed: 23856922.

11. Lax A, Sanchez-Mas J, Asensio-Lopez MC, et al. Mineralocorticoid receptor antagonists modulate galectin-3 and interleukin-33/ST2 signaling in left ventricular systolic dysfunction after acute myocardial infarction. JACC Heart Fail. 2015; 3(1): 50-58, doi: 10.1016/j.jchf.2014.07.015, indexed in Pubmed: 25458175.

12. Sánchez-Más J, Lax A, Asensio-López MD, et al. Modulation of IL-33/ST2 system in postinfarction heart failure: correlation with cardiac remodelling markers. Eur J Clin Invest. 2014; 44(7): 643-651, doi: 10.1111/eci.12282, indexed in Pubmed: 24837094.

13. Litwin SE, Katz SE, Morgan JP, et al. Serial echocardiographic assessment of left ventricular geometry and function after large myocardial infarction in the rat. Circulation. 1994; 89(1): 345-354, indexed in Pubmed: 8281668.

14. Xia Jg, Xu Ff, Qu Y, et al. Atorvastatin post-conditioning attenuates myocardial ischemia reperfusion injury via inhibiting endoplasmic reticulum stress-related apoptosis. Shock. 2014; 42(4): 365-371, doi: 10.1097/SHK.0000000000000224, indexed in Pubmed: 25004060.

15. Wolfrum S, Dendorfer A, Schutt M, et al. Simvastatin acutely reduces myocardial reperfusion injury in vivo by activating the phosphatidylinositide 3-kinase/Akt pathway. J Cardiovasc Pharmacol. 2004; 44(3): 348-355, indexed in Pubmed: 15475833.
16. Armstrong C. ACCF and AHA Release Guidelines on the Management of Heart Failure. Am Fam Physician. 2014; 90(3): 186-189, indexed in Pubmed: 25077725.

17. Mair J, Jaffe A, Apple F, et al. Cardiac biomarkers. Dis Markers. 2015; 2015: 370569, doi: 10.1155/2015/370569, indexed in Pubmed: 25960591.

18. Daniels LB, Bayes-Genis A. Using ST2 in cardiovascular patients: a review. Future Cardiol. 2014; 10(4): 525-539, doi: 10.2217/fca.14.36, indexed in Pubmed: 25301315.

19. Yancy CW, Jessup M, Bozkurt B, et al. 2013 ACCF/AHA guideline for the management of heart failure: executive summary: a report of the American College of Cardiology Foundation/American Heart Association Task Force on practice guidelines. Circulation. 2013; 128(16): 1810-1852, doi: 10.1161/CIR.0b013e31829e8807, indexed in Pubmed: 23741057.

20. Gaggin HK, Motiwala S, Bhardwaj A, et al. Soluble concentrations of the interleukin receptor family member ST2 and $\beta$-blocker therapy in chronic heart failure. Circ Heart Fail. 2013; 6(6): 1206-1213, doi: 10.1161/CIRCHEARTFAILURE.113.000457, indexed in Pubmed: 24114865.

21. Chatterjee S, Udell JA, Sardar P, et al. Comparable benefit of $\beta$-blocker therapy in heart failure across regions of the world: metaanalysis of randomized clinical trials. Can J Cardiol. 2014; 30(8): 898-903, doi: 10.1016/j.cjca.2014.03.012, indexed in Pubmed: 24939477.

22. Wikstrand J, Wedel H, Castagno D, et al. The large-scale placebocontrolled beta-blocker studies in systolic heart failure revisited: results from CIBIS-II, COPERNICUS and SENIORS-SHF compared with stratified subsets from MERIT-HF. J Intern Med. 2014; 275(2): 134-143, doi: 10.1111/joim.12141, indexed in Pubmed: 24118421.

23. Breidthardt $\mathrm{T}$, Balmelli $\mathrm{C}$, Twerenbold $\mathrm{R}$, et al. Heart failure therapy-induced early ST2 changes may offer long-term therapy guidance. J Card Fail. 2013; 19(12): 821-828, doi: 10.1016/j.cardfail.2013.11.003, indexed in Pubmed: 24239955.

24. Nguyen T, Shaheed A, Venigalla S, et al. Using beta-blockers to treat heart failure. JAAPA. 2014; 27(12): 50-55, doi: 10.1097/01. JAA.0000450813.21330.ee, indexed in Pubmed: 25417667.

25. Prijic S, Buchhorn R. Mechanisms of beta-blockers action in patients with heart failure. Rev Recent Clin Trials. 2014; 9(2): 58-60, indexed in Pubmed: 25198736.

26. Bølling R, Scheller NM, Køber L, et al. Comparison of the clinical outcome of different beta-blockers in heart failure patients: a retrospective nationwide cohort study. Eur J Heart Fail. 2014; 16(6): 678-684, doi: 10.1002/ejhf.81, indexed in Pubmed: 24706485. 